\title{
Optimal Control of a Continuous Distillation Process under Probabilistic Constraints
}

\author{
René Henrion ${ }^{1}, \mathrm{Pu} \mathrm{Li}^{2}$, Andris Möller ${ }^{1}$, Moritz Wendt ${ }^{2}$, and Günter Wozny ${ }^{2}$ \\ 1 Weierstraß-Institut für Angewandte Analysis und Stochastik (WIAS), Berlin, Germany \\ 2 Institut für Prozess- und Anlagentechnik, Technische Universität Berlin, Germany
}

\begin{abstract}
A continuous distillation process with random inflow rate is considered. The aim is to find a control (feed rate, heat supply, reflux rate) which is optimal with respect to energy consumption and which is robust at the same time with respect to the stochastic level constraints in the feed tank. The solution approach is based on the formulation of probabilistic constraints. An overall model including the dynamics of the distillation process and probabilistic constraints under different assumptions on the randomness of inflow is developed and numerical results are presented.
\end{abstract}

\section{INTRODUCTION}

As noted in [9], Section 1.2, continuous distillation processes are frequently characterized by uncertainties of their inflow. These may relate to the flow rate, to the composition of the mixture to be separated or to its temperature. Typically, the uncertainties are not completely irregular but follow a certain pattern caused by the operation of upstream units. Then it makes sense to model uncertainty as a stochastic parameter, the distribution of which can be estimated from history but the realization of which in the coming period of optimization is unknown. In the following, we are going to consider the rate of inflow as the only random parameter. As a consequence of possible unpredictable peaks, the inflow cannot be processed immediately but has to be stored in a feed tank before being directed at a controlled rate to the distillation unit (see [9], Figure 1). For technological reasons, one has to impose upper and lower level constraints for the feed tank preventing it from running full or empty. Both cases would require unpleasant compensating actions which are desirable to avoid (see [9], Section 1.3). Therefore, a problem will be formulated which reflects the objective to find a feed control being robust with respect to level constraints yet optimal in the sense of minimum energy consumption subject to product specifications.

From the stochastic nature of the inflow rate it is clear that the level constraints are stochastic too, and there is a choice to apply any of the methods briefly sketched in [9]. As costs of compensating actions for possible level violations are difficult to model on the one hand and a worst case approach is much too conservative or even impossible on the other hand, it is proposed to rely on probabilistic constraints. The gain over simply using typical profiles (or expected values) for the inflow rate will be illustrated later on. The aim of the subsequent analysis is to present a model of optimal control for continuous distillation with probabilistic feed tank constraints 
and to present numerical results for different assumptions on the randomness of inflow rate. As an example serves the separation of a methanol/water mixture.

\section{THE DisTILLATION PROCESS}

\subsection{Process description}

The process considered relates to a distillation column, as shown in Figure 1, for separating a binary mixture of water and methanol. The column has a diameter of $100 \mathrm{~mm}$ and 20 bubble-cap trays with a central downcomer. Isolation coat is mounted to reduce the heat loss from the column wall. The boilup is provided by an electrical thermal device, while the condensation is carried out by a total condenser with cooling water. The plant is equipped with temperature, pressure, level and flow rate measurements and electrical valves for the flow control. All input/output signals are treated by a process control system. Several control loops have been configured and implemented on the plant. The control system is connected to the local area network to manage experimental data. The composition of the feed stream, distillate and bottom product will be measured off-line by gas chromatography.

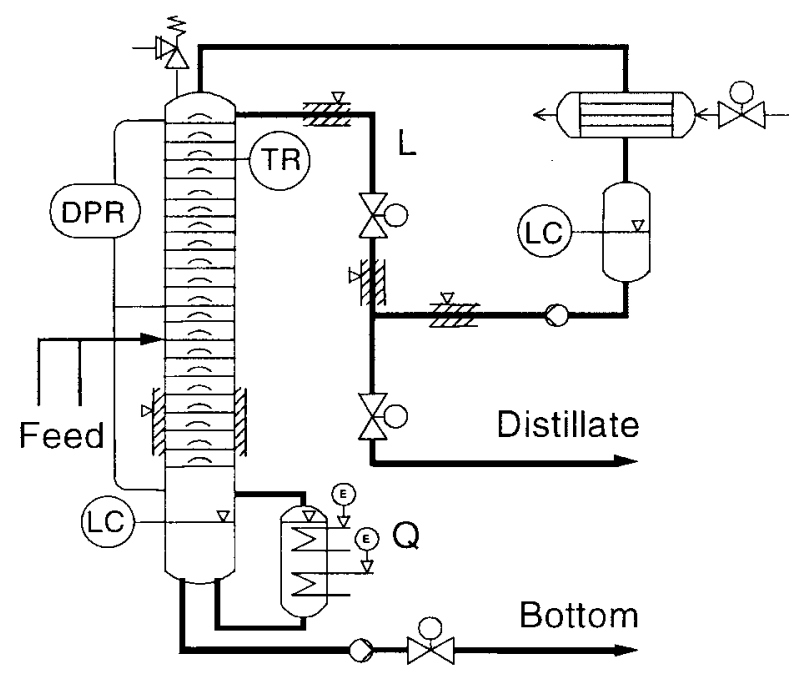

Figure 1. A pilot plant for distillation

From a feed tank, the feed stream with a given composition is fed to the column. The column is operated with atmospheric pressure. Operation of the plant means to run the column for keeping the desired distillate and bottom product purity under an uncertain feed flow profile. This leads to a control problem. Conventionally, to 
carry out this task two control loops have been used to manipulate the reflux rate and the reboiler duty. However, due to the changing feed stream, the operating point will also be changed frequently. Since distillation is a nonlinear dynamic process, the conventional control loops can not follow the desired changing operating point. Thus a conservative setpoint value, which is higher than the purity specifications, has to be used. But a conservative operation leads to more energy consumption than necessary, since the energy requirement for a column operation increases sensitively to the product purity, especially for a high purity distillation. Therefore, we propose to use a stochastic optimization approach to solve the column operation problem. This has the advantage over conventional feedback control that it employs the nonlinear rigorous model and includes uncertainties.

\subsection{The Model of Dynamics}

The dynamics of the distillation process are summarized in Table 1. Here we use a rigorous model details of which can be found in $[1,11,15]$. In the table $i=1, \ldots, I$ and $j=1, \ldots, J$ are the indices of components and trays (counting from the condenser over the internal trays to the reboiler), respectively. The control variables are the feed flow rate F, the reboiler duty $\mathrm{Q}$ and the reflux rate from the condenser $\mathrm{L}^{1}$. The dependent variables on each tray are the vapor and liquid mole fractions $Y_{i}^{j}, X_{i}^{j}$, vapor and liquid flow rate $V^{j}, L^{j}$, molar liquid holdup $M^{j}$, temperature $T^{j}$ and pressure $P^{j}$. The Murphree tray efficiency $\eta^{j}$ is included in order to describe the nonequilibrium behavior. The inclusion of the tray hydraulics is necessary to arrive at an index-one DAE system [3]. Additionally, it reflects the reality much better than other commonly used models, where molar or volumetric tray holdups and pressure drops are fixed. Really, the expressions containing $F$ are not included in the differential equations for all internal trays but only for the feed tray. The wet pressure drop $\phi_{w}$ is not applied to the tray 2 at the column head.

The DAE system also includes column specific parameters, applicable only to the individual construction and design of the pilot plant. The values of those parameters are listed in Table 2.

\subsection{Model Validation}

Before carrying out the optimization a model validation has to be made to ensure the accuracy of the computational results. In the model, the parameters of the vapour liquid equilibrium are taken from [6] while the parameters of the pure components are taken from [14]. The parameters for the tray holdup and the pressure drop calculated by the gas and liquid fluid dynamics are based on the tray geometric sizes. Several parameters (coefficients) in the model are strongly dependent on the column structure and the operating point and thus should be matched to the experimental results. We consider the tray efficiency in the rectifying section $\eta_{R}$ and stripping section $\eta_{S}$ of the column which are the most important parameters for the model validation. The weir constant $C_{w}$, a parameter in the Francis weir equation needed 


\section{Reboiler}

total mass balance:

$$
\frac{d}{d t}\left(M^{J}\right)=L^{J-1}-V^{J}-B
$$

component balance:

$\frac{d}{d t}\left(M^{J} X_{1}^{J}\right)=L^{J-1} X_{1}^{J-1}-V^{J} Y_{1}^{J}-B X_{1}^{J}$

energy balance:

$\frac{\mathrm{d}}{\mathrm{dt}}\left(\mathrm{M}^{\mathrm{J}} \mathrm{H}^{\mathrm{L}, \mathrm{J}}\right)=\mathrm{L}^{\mathrm{J}-1} \mathrm{H}^{\mathrm{L}, \mathrm{J}-1}-\mathrm{V}^{\mathrm{J}} \mathrm{H}^{\mathrm{V}, \mathrm{J}}-\mathrm{B} \mathrm{H}^{\mathrm{L}, \mathrm{J}}+\mathrm{Q}$

enthalpy calculation:

sum balances:

$$
H^{L, J}=\sum_{i=1}^{2} X_{i}^{J} h_{i}^{L}\left(T^{J}\right) \quad H^{V, J}=\sum_{i=1}^{2} Y_{i}^{J} h_{i}^{V}\left(T^{J}\right)
$$

$$
\sum_{i=1}^{2} X_{i}^{J}=1 \quad \sum_{i=1}^{2} Y_{i}^{J}=1
$$

vapor liquid equilibrium:

$$
Y_{i}^{J}=K_{i}\left(T^{J}, P^{I}, X_{1}^{J}, X_{2}^{J}\right) X_{i}^{J} \quad i=1,2
$$

pressure drop equation:

$\mathrm{P}^{\mathrm{J}-1}-\mathrm{P}^{\mathrm{J}}=\varphi_{\mathrm{d}}\left(\mathrm{V}^{\mathrm{J}}, \mathrm{T}^{\mathrm{J}}, \mathrm{Y}_{1}^{\mathrm{J}}, \mathrm{Y}_{2}^{\mathrm{J}}\right)+\varphi_{w}\left(\mathrm{M}^{\mathrm{J}-1}, \mathrm{~T}^{\mathrm{J}-1}, \mathrm{X}_{1}^{\mathrm{J}-1}, \mathrm{X}_{2}^{\mathrm{J}-1}\right)$

total mass balance:

\section{Internal Trays}

$$
\frac{d}{d t}\left(M^{j}\right)=F+L^{j-1}+V^{j+1}-L^{j}-V^{j}
$$

component balance:

$\frac{\mathrm{d}}{\mathrm{dt}}\left(M^{j} X_{1}^{j}\right)=F Z_{1}+\mathrm{L}^{j-1} X_{1}^{j-1}+\mathrm{V}^{\mathrm{j}+1} Y_{1}^{j+1}-\mathrm{L}^{j} X_{1}^{j}-\mathrm{V}^{j} Y_{1}^{j}$

energy balance:

$\frac{d}{d t}\left(M^{j} H^{L, j}\right)=F H^{F}+L^{j-1} H^{L, j-1}+V^{j+1} H^{V, j+1}-L^{j} H^{L, j}-V^{j} H^{V, j}$

enthalpy calculation:

$$
H^{L, j}=\sum_{i=1}^{2} X_{i}^{j} h_{i}^{L}\left(T^{j}\right) \quad H^{V, j}=\sum_{i=1}^{2} Y_{i}^{j} h_{i}^{V}\left(T^{j}\right)
$$

sum balances:

$$
\sum_{i=1}^{2} X_{i}^{j}=1 \quad \sum_{i=1}^{2} Y_{i}^{j}=1
$$

vapor liquid equilibrium:

$$
Y_{i}^{j}=\eta^{j} K_{i}\left(T^{j}, P^{j}, X_{1}^{j}, X_{2}^{j}\right) X_{i}^{j}+\left(1-\eta^{j}\right) Y_{i}^{j+1} \quad i=1,2
$$

Francis weir equation:

$$
L^{j}=\psi\left(M^{j}, T^{j}, X_{1}^{j}, X_{2}^{J}\right)
$$

pressure drop equation:

$$
\mathrm{P}^{\mathrm{j}-1}-\mathrm{P}^{\mathrm{j}}=\varphi_{\mathrm{d}}\left(\mathrm{V}^{\mathrm{j}}, \mathrm{T}^{\mathrm{j}}, \mathrm{Y}_{1}^{\mathrm{j}}, \mathrm{Y}_{2}^{\mathrm{j}}\right)+\varphi_{w}\left(\mathrm{M}^{\mathrm{j}-1}, \mathrm{~T}^{\mathrm{j}-1}, \mathrm{X}_{1}^{\mathrm{j}-1}, \mathrm{X}_{2}^{\mathrm{j}-1}\right)
$$

\section{Total Condenser}

total mass balance:

$$
\frac{\mathrm{d}}{\mathrm{dt}}\left(\mathrm{M}^{1}\right)=\mathrm{V}^{2}-\mathrm{L}^{1}-\mathrm{D}
$$

component balance:

$\frac{\mathrm{d}}{\mathrm{dt}}\left(M^{1} \mathrm{X}_{1}^{\mathrm{L}, 1}\right)=\mathrm{V}^{2} \mathrm{X}_{1}^{\mathrm{V}, 2}-\mathrm{L}^{1} \mathrm{X}_{1}^{\mathrm{L}, 1}-\mathrm{D} \mathrm{X}_{1}^{\mathrm{L}, 1}$

enthalpy calculation:

$$
H^{L, 1}=\sum_{i=1}^{2} X_{i}^{1} h_{i}^{L}\left(T^{1}\right) \quad\left(T^{1}=\text { const. }\right)
$$

Table 1. Dynamics of the distillation process 


\begin{tabular}{|c|c|c|c|}
\hline \multicolumn{4}{|c|}{ Construction Parameters } \\
\hline $\mathrm{N}$ & Number of trays & 20 & - \\
\hline$A$ & Tray area & 0.008992 & $\mathrm{~m}^{2}$ \\
\hline$A_{F}$ & Free area & 0.00045 & $\mathrm{~m}^{2}$ \\
\hline$l_{W}$ & Weir length & 0.015 & $\mathrm{~m}$ \\
\hline$h_{W}$ & Weir height & 0.03 & $\mathrm{~m}$ \\
\hline \multicolumn{4}{|c|}{ Operation Parameters } \\
\hline$\varepsilon$ & Vol. fraction of liquid in the bubble area & 0.5 & - \\
\hline $\mathrm{T}_{\mathrm{R}}$ & Reflux Temp. & 60 & ${ }^{\circ} \mathrm{C}$ \\
\hline $\mathrm{T}_{\mathrm{F}}$ & Feed Temp. & 60 & ${ }^{\circ} \mathrm{C}$ \\
\hline$V_{D}$ & Vol. of Dist. Vessel & 1.5 & $\mathrm{~m}^{3}$ \\
\hline$V_{B}$ & Vol. of bottom Vessel & 6.0 & $\mathrm{~m}^{3}$ \\
\hline $\mathrm{N}_{\mathrm{F}}$ & Feed tray & 14 & - \\
\hline
\end{tabular}

Table 2. Column specific data

for describing the dynamic behavior of the column is also estimated from experimental data. The friction factor $\zeta_{w}$, a parameter in the pressure drop equation used for calculating the dry pressure loss, has to be verified as well. Moreover, the total heat loss $\mathrm{Q}_{V}$ from the column, reboiler and the pipelines has been found at a level of about $10 \%$ of the reboiler duty. This result is important for the implementation of the computed reboiler duty values $Q_{e f f}$ on the real plant.

To accomplish the above verifications, 10 steady-state operating points have been gained through experiment. The parameters are adjusted by comparing the measured data (temperature, pressure as well as pressure drop, flow rates, compositions and reboiler duty) with the simulation results for these operating points. The comparison was evaluated by the least square function related to the temperature profiles. Moreover,the measured data of the dry pressure drop of the rectifying section and the total pressure drop of the stripping section during the startup phase were also used for estimating the parameters $\zeta_{w}$ and $C_{w}$. finally, the adjusted values for those parameters are $\eta_{R}=0.85, \eta_{S}=0.80, C_{w}=7.614, \zeta_{w}=2.3046$, $\mathrm{Q}_{V}=0.6 \mathrm{KW}$.

To verify these values two tests were done by step changes of the feed rate, reflux rate and reboiler duty from a steady-state operating point of the real plant. Figure 2 (left) shows the temperature responses along the column in the first run, where the black lines with noises are the measured curves and the grey lines are the simulated results. It can be seen that a very good agreement has been received for the top and bottom temperature. The temperature on tray 17 and 18, which are the two most sensitive trays in the column, has a 2-3 C difference between the measured and computed results. The reason for this is the existence of slight disturbances from the atmospheric pressure and temperature during the experiment which led to the oscillation of the column pressure and heat loss from the column. The fact that 
both the measured and simulated curves have the same tendency demonstrates that the inclusion of the hydraulic computation leads to a successful description of the dynamic behavior for the column operation. The second test was made for a step change in the opposite direction. The temperature profiles are shown in Figure 2 (right). It can be noted that the temperature on the trays in the rectifying section is not sensitive to the step changes, especially for the top of the column. But the temperature in the stripping section has a significant sensitivity. This phenomenon can be identified from the form of the $x$-y-diagram of the methanol-water mixture.
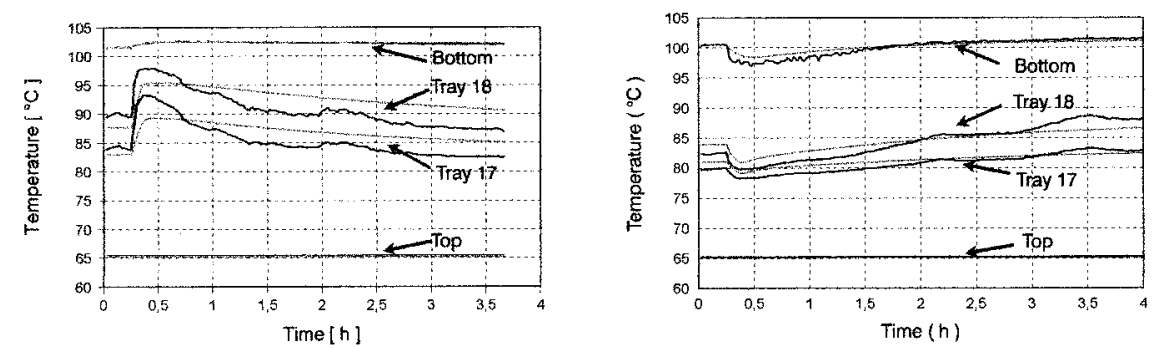

Figure 2. Simulated and measured temperature profiles: experiment 1 (left) and 2 (right)

\subsection{Relation between Feed Rate and Reboiler Duty}

Often one observes a tight relation between the feed supply and the required energy (reboiler duty). In general (especially for dynamic processes), however, this relation is not strong enough to substitute one of these control variables by the others which would lead to simpler mathematical models. In particular, the consideration of probabilistic constraints to be described below could be separated then from the dynamics of the distillation process. The following computational results demonstrate that the total energy consumption (our goal function) is not only a function of the total amount of feed but also of the shape of the feed rate profile.

Figure 3 shows two profiles for the feed flow rate having extremely different shapes. If the required purity constraints are exactly satisfied (here: 0.995 for the distillate and bottom products) during the time horizon, the theoretical energy consumption in the two cases should have almost the same value. It turns out, however, that the total energy consumption for the smooth profile is less than for the irregular profile at a factor of around 0.97 . The reason can be figured out from the respective purity profiles in Figure 4: The purity constraints can be almost exactly satisfied for the smooth feed trajectory, whereas in the second case a higher purity than required has to be realized to ensure the constraints to be held under the drastically changing 


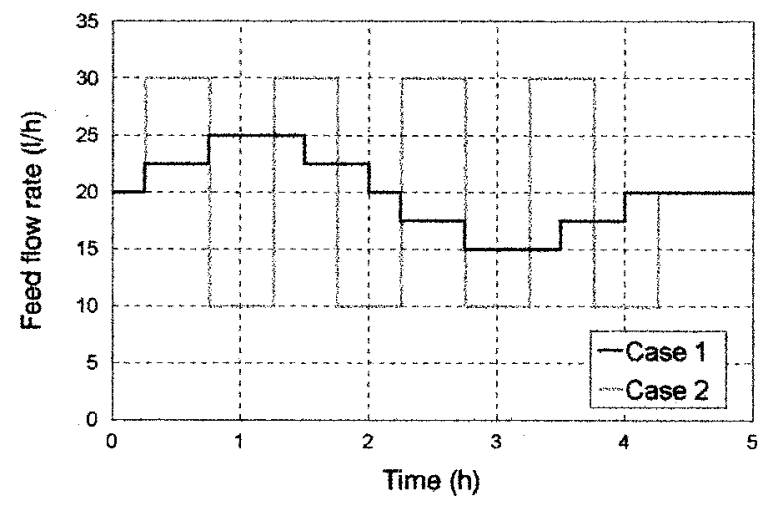

Figure 3. Two different profiles for the feed flow rate

feed stream. This behavior of the purity profiles results from the piecewise constant profiles of the control functions on the discretization scheme.
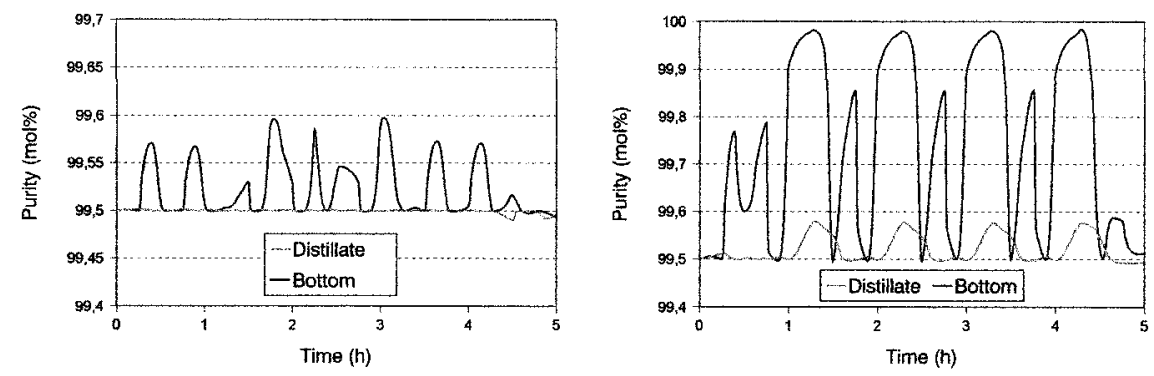

Figure 4. Optimal purity profiles for the feed trajectory from Figure 3: smooth case (left) and irregular case (right)

\section{Probabilistic Feed Tank Constraints}

We assume that our optimization horizon is given by the interval $\left[t_{0}, t_{1}\right]$. As mentioned in the introduction, the filling level $l(t)$ of the feed tank has to satisfy the simple constraints

$$
l^{\min } \leq l(t) \leq l^{\max } \quad \text { for all } t \in\left[t_{0}, t_{1}\right] .
$$

In view of the contradiction between the finite optimization horizon and the infinite nature of our continuous distillation process, one has to impose an additional so- 
called cycling constraint on the filling level. Otherwise, an optimal strategy with respect to energy consumption would certainly consist in running the unit on the lowest possible feed extraction level. This would result in a high filling level $l\left(t_{1}\right)$ at the end of the optimization horizon. If, for instance, $l\left(t_{1}\right)=l^{\max }$, then it may well happen that there does not exist any feasible control of feed extraction for the next horizon $\left[t_{1}, t_{2}\right]$ due to the risk of violating the upper level constraint (when the inflow runs faster than the maximum possible rate of feed extraction). In other words, one has to make sure that optimization during any time period is not done at the expense of coming periods. This can be realized by the requirement, that all inflow has to be processed over the interval, or in equivalent terms, the final and initial filling levels coincide: $l\left(t_{1}\right)=l\left(t_{0}\right)$. For a higher operational flexibility it is reasonable to formulate the following slight generalization of the last constraint:

$$
l\left(t_{1}\right)=l^{\prime}
$$

Here, $l^{\prime} \in\left[l^{\min }, l^{\max }\right]$ is any pre-defined end-level which may be in the middle of $l^{\min }$ and $l^{\max }$ in the regular case, but which could also be appropriate to choose closer to $l^{\max }$, for instance, in front of a week-end where no or few amounts of inflows only can be expected.

Next, we are going to take into account the random character of the filling level caused by the stochastic nature of inflows. To this aim, denote by $\xi$ the inflow rate which we assume to be a one-dimensional stochastic process. Now, the filling level as a function of inflow rate $\xi$, of feed extraction rate $F$ and of time $t$ writes as

$$
l(\xi, F, t)=l_{0}+\int_{t_{0}}^{t}(\xi(\tau)-F(\tau)) d \tau .
$$

Here, $l_{0}$ denotes the initial level at $t_{0}$. Accordingly, (1) and (2) turn into uncertain constraints of the type

$$
l^{\min } \leq l(\xi, F, t) \leq l^{\max } \quad \text { for all } t \in\left[t_{0}, t_{1}\right] \quad \text { and } l\left(\xi, F, t_{1}\right)=l^{\prime} .
$$

According to [9], uncertain constraints can be turned into implementable constraints by expected value substitution or by probabilistic constraints. Taking expected values seems to be justified for the cycling constraint, since violations of (3) by single inflow realizations do not cause serious harms and we may content ourselves with the fact that over many repeated optimization periods there will be no systematic gain on average by violating (3) in a specific period at the expense of later periods. Accordingly, (2) becomes

$$
l\left(\mathbb{E} \xi, F, t_{1}\right)=l^{\prime},
$$

where ' $\mathbb{E}$ ' denotes expectation. On the other hand, frequent violations of the level constraints (1), as they have to be expected when feasibility is reached only for an average profile of inflow rate, do cause troubles for the compensation of which is difficult to measure in terms of costs. Consequently, one may be interested in controls $F$ being feasible with high probability according to the distribution of $\xi$ :

$$
P\left(l^{\min } \leq l(\xi, F, t) \leq l^{\max } \quad \text { for all } t \in\left[t_{0}, t_{1}\right]\right) \geq p .
$$


Here, $P$ refers to the probability measure associated with $\xi$ and $p \in(0,1)$ is some probability level at which we require the inequalities inside parentheses to be fulfilled. According to [9], Section 4.1 there is much degree of modelling freedom concerning a joint or individual treatment of probabilistic constraints. We shall focus on a formulation in which time dependence appears as joint constraints whereas lower and upper levels are considered individually. More precisely, the following two probabilistic constraints shall be imposed with respect to filling levels of the feed tank:

$$
\begin{array}{ll}
P\left(l^{\min } \leq l(\xi, F, t)\right. & \text { for all } \left.t \in\left[t_{0}, t_{1}\right]\right) \geq p \\
P\left(l^{\max } \geq l(\xi, F, t)\right. & \text { for all } \left.t \in\left[t_{0}, t_{1}\right]\right) \geq p .
\end{array}
$$

\section{The Overall Problem and its Numerical Solution}

Combining the model of dynamics described in section 2.2 and the stochastic feed tank constraints, we are now in a position to set up our control problem in the following compact way:

$$
\begin{aligned}
& \min \mathrm{J}(\mathrm{u}) \\
\text { subject to } & \\
\dot{x} & =\mathrm{f}(\mathrm{x}, \mathrm{y}, \mathrm{u}) \\
\mathrm{g}(\mathrm{x}, \mathrm{y}) & =0 \\
x\left(\mathrm{t}_{0}\right) & =x_{0} \\
\mathrm{l}\left(\mathbb{E} \xi, \mathrm{u}, \mathrm{t}_{1}\right) & =\mathrm{l}^{\prime} \\
\mathrm{P}\left(\mathrm{l}^{\min } \leq \mathrm{l}(\xi, \mathrm{u}, \mathrm{t}) \quad \forall \mathrm{t} \in\left[\mathrm{t}_{0}, \mathrm{t}_{1}\right]\right) & \geq \mathrm{p} \\
\mathrm{P}\left(\mathrm{l}^{\max } \geq \mathrm{l}(\xi, \mathrm{u}, \mathrm{t}) \quad \forall \mathrm{t} \in\left[\mathrm{t}_{0}, \mathrm{t}_{1}\right]\right) & \geq \mathrm{p} \\
\mathrm{u} \in \mathrm{U}, \mathrm{x} \in \mathrm{X}, \mathrm{y} \in \mathrm{Y} &
\end{aligned}
$$

Here, $u, x, y, \xi$ are functions defined on our optimization interval $\left[t_{0}, t_{1}\right]$ and representing control, differential state, algebraic state and random variables, respectively. For instance, $u=\left(F, Q, L^{1}\right)$ comprises the extraction rates for feed, heat supply and reflux rate. The state variables consist of all the remaining physical quantities in the dynamics of the distillation system. Finally, $\xi$ refers to the stochastic inflow rate. The goal function to be minimized is the total heat consumption

$$
J(u)=\int_{t_{0}}^{t_{1}} Q(t) d t .
$$

We assume that the condenser cooling duty is negligible since it will not influence our solution approach and, furthermore, it depends on the specific circumstances whether or not additional costs arise by cooling.

The first three equalities in the constraints above contain the differential and algebraic equations of the dynamics as well as initial conditions. The following 
three inequalities relate to the cycling constraint (3) and to the probabilistic feed level constraints (5). The abstract constraints at the end represent simple bounds on the control and state variables; in particular, $u \in U$ incorporates limitations on the feed rate, heat supply and flow rates for bottom and distillate products, $x \in X$ includes purity conditions on the bottom and distillate products as well as constraints for the holdup in the reboiler and condenser while $y \in Y$ defines bounds on physical quantities like temperature, pressure etc.

A common approach for solving control problems like $(\mathrm{P})$ is the so-called direct method [2,13,16-18], where the differential algebraic equations are discretized and the corresponding functions are finitely parametrized in order to yield a (possibly large scale) nonlinear optimization problem in finite dimensions. In our case, it may be written as

$$
\begin{aligned}
& \min \varphi(u) \quad(N L P) \\
& \text { subject to } \\
& \mathrm{G}(x, y, u)=0 \\
& h_{1}(\mathbb{E} \xi, u)=l^{\prime} \\
& P\left(h_{2}(\xi, u) \leq 0\right) \geq p \\
& P\left(h_{3}(\xi, u) \leq 0\right) \geq p \\
& u \in U, x \in X, y \in Y
\end{aligned}
$$

where now $u, x, y, \xi$ are finite-dimensional vectors.

A direct simultaneous approach based on collocation will be used here to discretize the differential algebraic equation (DAE). The obtained optimization problem is then solved by the SQP method SNOPT [4], [5]. From technological requirements, the controls have to be piecewise constant on a given grid here. For instance, the operator of the system may be able to only tune constant values once an hour. The grid for the DAE is chosen equal to or as a refinement of the grid for the controls to prevent that a jump of the controls is within an integration interval of the DAE. On each subinterval of the refined grid the DAE is treated by a 3 stage Radau IIa (collocation) scheme [7]. The resulting collocation conditions are formulated in terms of the algebraic variables and in terms of the derivatives of the differential state variables at the collocation points $[16,17]$. Additional conditions are needed to ensure continuity of the state variables between the end and the beginning of two succeeding collocation intervals. For consistency purposes these conditions can not be imposed on all variables but only for a part of the variables depending on the index. Since the differential index of our DAE is 1 , it is sufficient to restrict the continuity condition to the differential state variables [12]. That means that for the whole problem as well as for the integration intervals initial values are given only for the differential state variables. In convergence, consistency is then automatically fullfiled since the Radau IIa scheme includes the endpoint but excludes the initial point of each collocation interval and for given differential state variables the algebraic variables are uniquely determined by the algebraic constraints of the Index-1-DAE. 
In order to solve the overall problem, one has to incorporate numerical techniques for dealing with the probabilistic constraints into the framework described before. The concrete realization depends on the structure of probabilistic constraints as it results from different modelling assumptions. For instance, assuming $\xi$ originally to represent a Gaussian process, the treatment of constraints (5) leads to the evaluation of multivariate normal distribution functions after passing to a time discretization. For implementation, we linked Szantai's simulation scheme described in [9], Section 4.3 and realized in his code BERNOR to the SQP code SNOPT. As a preliminary step of numerical solution, probability maximization was carried out (see [9], Section 4.4).

\section{Stochastic Models For the Inflow RATES}

We shall consider two basically different models for the stochastic inflow rate: a model describing some elementary single process of inflow generation and a model reflecting the superposition of numerous such elementary processes. Both situations may be relevant in practice depending on the nature of production processes prior to distillation. We start with the model relating to a lot of independent elementary processes of equal structure. According to the law of large numbers, it is reasonable to assume that the rate $\xi_{t}$ of overall inflows is a Gaussian process, which means that each finite selection $\left(\xi_{\tau_{1}}, \ldots, \xi_{\tau_{n}}\right)$ of random variables with $\tau_{1}, \ldots, \tau_{n} \in$ $\left[t_{0}, t_{1}\right]$ has a multivariate normal distribution. The second model considers a fixed inflow function the realization of which takes place at a random starting time. The difference between both models is illustrated in Figure 5.
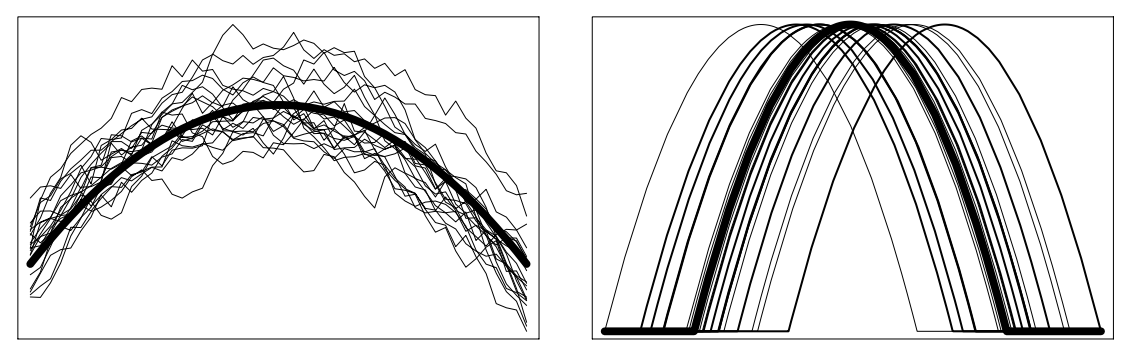

Figure 5. Comparison of two different stochastic models for the inflow rate. Left: Gaussian process; right: fixed profile with random initial time. Thick curves represent expected values of the corresponding processes and thin curves illustrate possible samples (observations) thereof 


\subsection{Inflow Rate with Multivariate Normal Distribution}

According to the discretized optimization problem (6) let $\tau_{0}<\cdots<\tau_{N}$ be a subdivision of the time interval $\left[t_{0}, t_{1}\right]$ with $\tau_{0}=t_{0}$ and $\tau_{N}=t_{1}$. For such fixed subdivision, we may identify the average inflow rate $\xi_{i}$ during the interval $\left[\tau_{i-1}, \tau_{i}\right]$ with the total amount of inflow over this period $(i=1, \ldots, N)$. According to the statement above, it is assumed that the $\mathrm{N}$ - dimensional random vector $\xi=\left(\xi_{1}, \ldots, \xi_{\mathrm{N}}\right)$ has a multivariate normal distribution, i.e., $\xi \sim \mathcal{N}(\mu, \Sigma)$, where $\mu$ and $\Sigma$ are the expectation and covariance matrix, respectively, of $\xi$. In the numerical experiments described below, we have supposed a covariance structure with decreasing positive values at increasing distance in time. In practice, $\mu$ and $\Sigma$ have to be estimated from observed data of the inflow process. In the first diagram of Figure 7 a set of sample paths of an exemplary inflow process is illustrated. Here, loosely speaking, the typical profile corresponds to $\mu$, whereas $\Sigma$ accounts for the scattering around $\mu$ and the smoothness of the sample paths.

In order to specify the functions $h_{1}, h_{2}, h_{3}$ in (NLP), we recall that our feed tank constraints are special cases of the storage level constraints introduced in [9], Section 4.2. Accordingly, the filling level in the feed tank at time $\tau_{i}$ calculates as

$$
l_{0}+\sum_{j=1}^{i} \xi_{j}-\sum_{j=1}^{i} F_{j}
$$

where $l_{0}$ is the filling level at $\tau_{0}=t_{0}$ and $\xi_{j}, F_{j}$ refer to the amounts of inflow and feed extraction, respectively during $\left[\tau_{j-1}, \tau_{j}\right]$. Now, the cycling constraint (3) requires that the expectation of the final filling level (at $\tau_{N}=t_{1}$ ) equals $l^{\prime}$. Taking into account that $\mathbb{E} \xi_{j}=\mu_{j}$, the cycling constraint writes as the following simple linear restriction in the control variable $\mathrm{F}$ :

$$
\sum_{j=1}^{N} F_{j}=l_{0}-l^{\prime}-\sum_{j=1}^{N} \mu_{j}
$$

Next, we turn to the first probabilistic constraint in (6) or (5), respectively (the second one being analogous). According to (7), one may establish this lower level restriction in discretized form as

$$
P\left(l^{\min } \leq l_{0}+\sum_{j=1}^{i} \xi_{j}-\sum_{j=1}^{i} F_{j} ; \quad i=1, \ldots, N\right) \geq p .
$$

After some transformations detailed in [10], this last relation can be equivalently written in the explicit form $\Phi(\alpha(F)) \geq p$, where $\alpha(F)$ is a simple affine linear mapping and $\Phi$ refers to a $\mathrm{N}$-dimensional standard normal distribution with suitable correlation matrix. Consequently, the whole issue of coping with probabilistic feed tank constraints hinges upon the ability of calculating distribution functions of multivariate normally distributed random vectors. Some possible approaches to do so have been presented in [9], Section 4.3. 


\subsection{Fixed Inflow Profiles with Stochastic Initial Time}

Next we turn to an inflow process with a deterministic profile which is realized at a random starting time. In a non-discretized setting, the profile is given by some nonnegative function $v \in \mathrm{L}_{+}^{\infty}([0, \mathrm{~d}])$ (determined, e.g., by a fixed operating pattern of some machine), where d denotes the duration of the inflow. In the simplest case, the profile is constant over $[0, d]$. Defining $\xi^{\prime}$ as the random initial time, the inflow process becomes

$$
\xi=\left\{\begin{array}{ll}
v\left(\mathrm{t}-\xi^{\prime}\right) & \text { if } \mathrm{t} \in\left[\xi^{\prime}, \xi^{\prime}+\mathrm{d}\right] \\
0 & \text { else }
\end{array} .\right.
$$

In [8] it is shown that the probabilistic constraints (5) can be transformed into the explicit linear (functional) constraints

$$
\varphi^{1}(\mathrm{t}) \leq \int_{\mathrm{t}_{0}}^{\mathrm{t}} \mathrm{F}(\tau) \mathrm{d} \tau \leq \varphi^{2}(\mathrm{t}) \quad \forall \mathrm{t} \in\left[\mathrm{t}_{0}, \mathrm{t}_{1}\right]
$$

for the feed extraction rate F. Here,

$$
\begin{aligned}
\varphi^{1(2)}(\mathrm{t}) & =\mathrm{l}_{0}-\mathrm{l}^{\max }\left(\mathrm{l}^{\min }\right)+\min \left\{\max \left\{0, \mathrm{~V}\left(\mathrm{t}-\mathrm{q}_{1}\left(\mathrm{q}_{2}\right)\right)\right\}, \mathrm{V}(\mathrm{d})\right\} \\
\mathrm{V}(\mathrm{t}) & =\int_{0}^{\mathrm{t}} v(\tau) \mathrm{d} \tau \\
\mathrm{q}_{1} & =\sup \left\{\mathrm{t} \mid \mathrm{P}\left(\xi^{\prime} \leq \mathrm{t}\right) \leq 1-\mathrm{p}\right\} \\
\mathrm{q}_{2} & =\inf \left\{\mathrm{t} \mid \mathrm{P}\left(\xi^{\prime} \leq \mathrm{t}\right) \geq \mathrm{p}\right\}
\end{aligned}
$$

This means that the constraining functions $\varphi^{1}, \varphi^{2}$ are easily obtained from the data of the problem. In particular, $q_{1}$ and $q_{2}$ are related with appropriate quantiles of the distribution of the random initial time $\xi^{\prime}$. In discretized form, one arrives at the linear restrictions

$$
\varphi^{1}\left(\tau_{i}\right) \leq \sum_{j=1}^{i} F_{j} \leq \varphi^{2}\left(\tau_{i}\right) \quad i=1, \ldots, N .
$$

It is worth mentioning (cf. [8, Thm. 5]) that exactly the same constraints result if, in contrast to (5), the probabilistic constraints are considered individually with respect to time, i.e.,

$$
P\left(l^{\min } \leq l(\xi, F, t)\right) \geq p \quad \text { and } \quad P\left(l^{\max } \geq l(\xi, F, t)\right) \geq p \quad \text { for all } t \in\left[t_{0}, t_{1}\right] .
$$

This means that the distinction between individual and joint constraints is no longer relevant in this specific model (see discussion in [9], Section 4.1).

\section{Numerical Results}

In the following, we describe numerical results obtained for the model developed above. A time horizon of 16 hours is considered. In order to illustrate the gain of using stochastic information, the results of models with probabilistic constraints shall 
be opposed to those obtained when simply assuming the expected inflow profile. We start with the model of a (discretized) Gaussian process for the inflow rate. The first diagram of Figure 7 shows 100 possible inflow realizations for an example. As a distillation unit, a column consisting of a reboiler, a total condenser and three internal trays, is considered. The required purities are defined by methanol concentrations of $\geq 80 \%$ and $\leq 10 \%$ in the condenser and reboiler, respectively.

If the calculations are based on the expected inflow profile then the solid curves in the first three diagrams of Figure 6 result as optimal - in the sense of minimum heat consumption - profiles for feed extraction rate, reflux rate and heat supply. Not surprisingly, heat consumption and feed extraction run almost in parallel. The last three diagrams illustrate the profiles of a selection of associated state variables, namely purity, pressure and liquid flow rate. The purity diagram confirms in particular the satisfaction of required purities in reboiler and condenser, respectively. These solutions are now contrasted with the solutions based on probabilistic feed tank constraints when imposing a probability level of $p=0.9$. The optimal profiles for control variables are represented by dotted curves in the first three diagrams of Figure 6. Although the differences appear to be negligible, they have a significant impact on the robustness of the process as shall be seen next. Corresponding plots of state variable profiles are similar to the previous ones and omitted here for the sake of brevity.

Assuming for a moment that the inflow profile realizes indeed its expected values, the filling levels plotted in the second diagram of Figure 7 result from applying the respective feed extraction controls of the first diagram of Figure 6. Again, the solid curve represents the solution based on the expected inflow profile whereas the dotted curve relates to probabilistic constraints. Both trajectories are feasible with respect to satisfying a filling level between the upper value of 1500 and the lower value of 500. Note that the lower level is attained by the solid curve after around 4 hours whereas the dotted curve remains slightly above. The starting level was supposed to be at 1000 and a cycling constraint was set up in order to realize the same level at the end of the time horizon which means that eventually all incoming substance was separated by the distillation column.

In practice, however, it is very unlikely that the expected value is realized. Rather, one is likely to observe one out of the 100 sample profiles plotted in the first diagram of Figure 7 which scatter more or less around the expected profile. Therefore, the filling level obtained from the optimal feed extraction profiles has to be verified with regard to these samples instead. The associated 100 profiles of filling levels are given in the third (application of the feed extraction control based on assuming the expected inflow profile) and fourth (application of the feed extraction control based on probabilistic constraints) diagrams of Figure 7. In both cases it becomes obvious how uncertainty evolves over time with ever increasing variances of filling levels. Furthermore, in both cases the average filling level at the end coincides with the initial value which illustrates satisfaction of the (stochastic) cycling constraint imposed in (3).

In contrast, and more important, a frequent violation of the lower level constraint can be observed in the first case. As this is hardly visible from the total diagram, the 
interesting section is zoomed in the diagrams below. Indeed, 51 out of the 100 samplesare found to violate the lower level at some time between three or five hours after initial time. This means that undesirable compensating actions have to be carried out with probability of around $50 \%$ upon choosing a feed extraction control based on assuming the expected inflow profile and thus ignoring information on the distribution of the random process. This result of $50 \%$ violations is not surprising if one recalls that in the expected case the lower level is exactly attained (solid curve in the second diagram of Figure 7).

In contrast, application of the feed extraction control based on probabilistic constraints yields significantly less violations of the lower level as can be easily verified from the corresponding zoomed plot again. This time, just 12 out of the 100 samples fall below $l^{\mathrm{min}}$ which is in good accordance with the chosen probability level of $p=0.9$. The upper level constraint (not zoomed) is violated for less than 10 samples in both cases. Summarizing, there is a considerable gain in robustness when passing from expected value solutions to probabilistic constraints solutions. The value of stochastic information is reflected by the probability of violation dropping from $50 \%$ to $10 \%$. In general, one would expect to obtain such gain in robustness only at the expense of worsened values of the objective function. However, there is no measurable difference in heat consumption for the two discussed controls here (compare area below curves of the respective diagram in Figure 6).

Now we turn to corresponding results when assuming the alternative stochastic model of a fixed inflow profile with random initial time. As a fixed profile, we consider an inflow process with constant rate and with a duration of 9 hours. The initial time is assumed to be uniformly distributed in the interval $[1,7]$. As mentioned above, any other bounded fixed profile and any other random distribution could be chosen equally well as long as the required quantiles are available.

Figure 8 shows the obtained results relating to feed tank constraints and omitting other plots related to the dynamics of the distillation process. In the first diagram, the optimal feed extraction rates are plotted with the meanings of the two curves being analogous to the discussion before. The two bottom diagrams represent the realized filling levels when applying the two different controls to 100 samples of the inflow process. Thick curves refer to the expected inflow. Again, the lower level is violated by almost one half of the samples for the expected value solution whereas just a few violations occur for the probabilistic constraints solution. In the right top diagram the violation probabilities are plotted as functions of time.

It can be seen that the expected value solution reaches a peak of around $45 \%$ in the period between 4 and 10 hours. This is in contrast to the probabilistic constraints solution with a peak of $5-10 \%$ (in good accordance with the level $p=0.9$ ) in the period between 6 and 8 hours. 

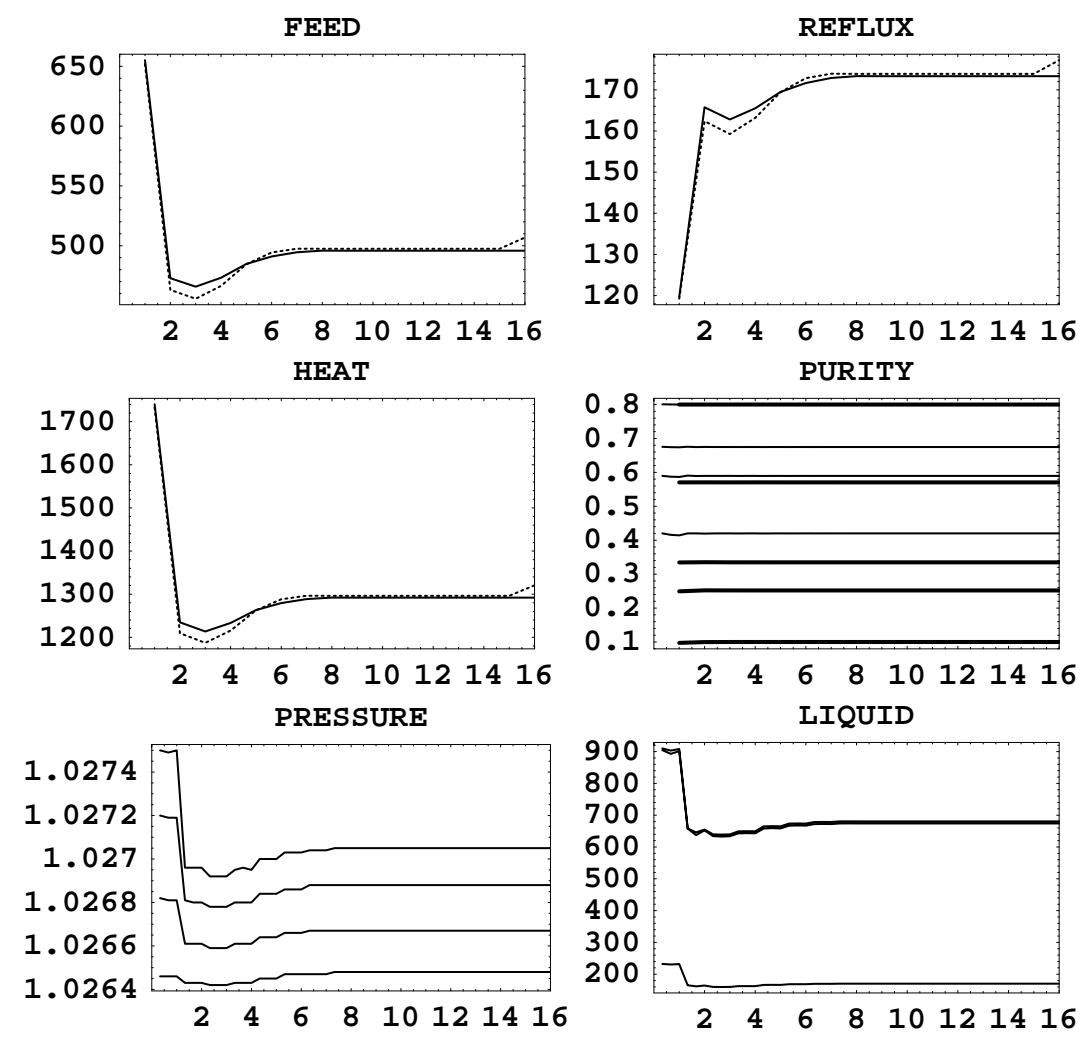

Figure 6. Results for the optimal control of continuous distillation with the inflow rate being a Gaussian process. The first three diagrams plot the optimal profiles for the three control variables (feed extraction rate, reflux and heat supply). The solid curves relate to the assumption of the expected inflow profile whereas the dotted curve relates to the model with probabilistic feed tank constraints. The last three diagrams illustrate the profiles of some selected state variables (purity, pressure, liquid flow rate). Different curves correspond to subsequent trays (possibly including reboiler and condenser) of the distillation column. The purity diagram distinguishes methanol concentrations in the liquid phase (thick lines) from those in the vapour phase (thin lines) 

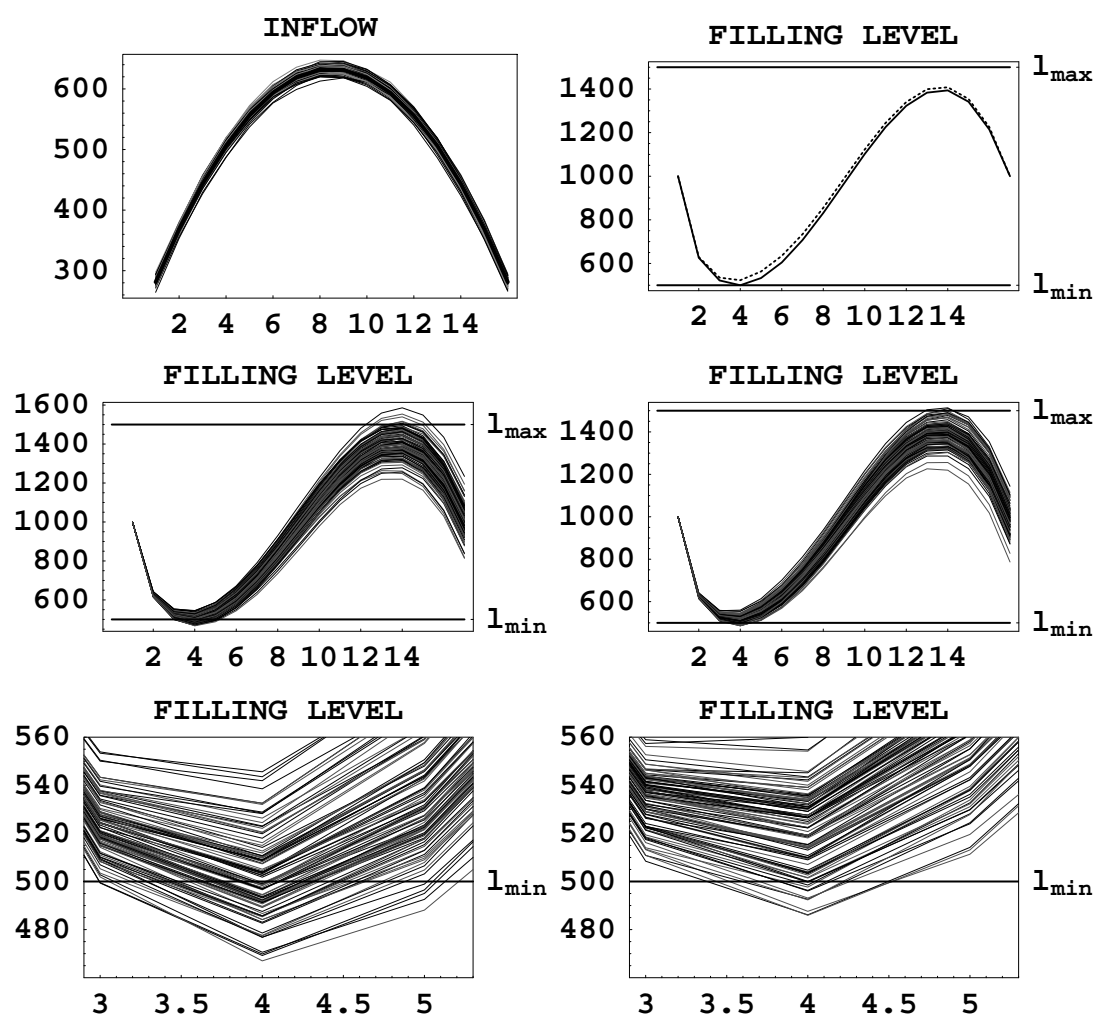

Figure 7. Filling levels in feed tank upon applying the optimal The first diagram illustrates 100 samples of a possible inflow process. In the second diagram, the filling levels in the feed tank are plotted under the assumption that the expected inflow profile is observed and the feed extraction profiles from Figure 6 are applied. The next two diagrams show the resulting filling levels for the 100 inflow samples of the first diagram. The left diagram relates to the expected value solution whereas the right one relates to the probabilistic constraints solution. The critical section with respect to lower level violation is zoomed in the respective diagrams at the bottom 


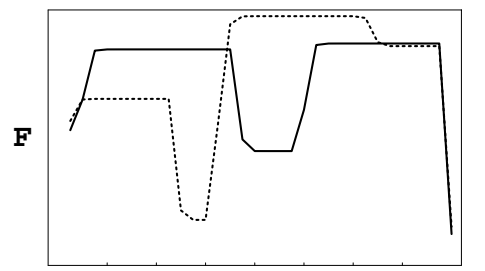

$2446 \quad 8 \quad 101214$

t

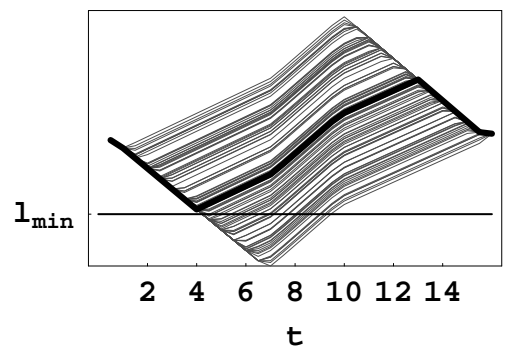

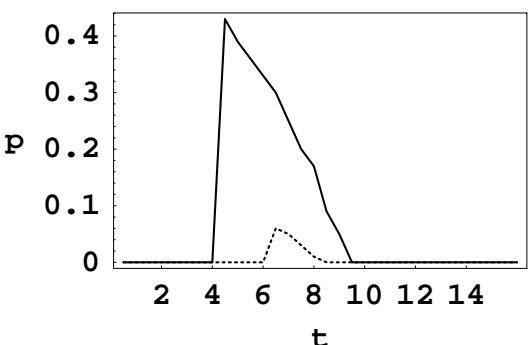

t

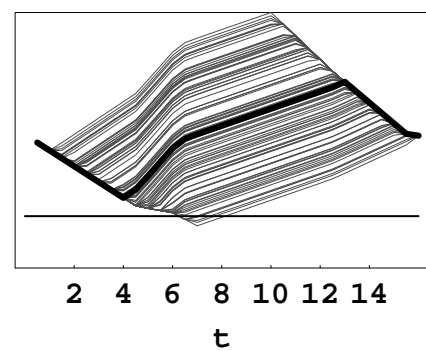

Figure 8. Results for the optimal control of continuous distillation with the inflow rate having a constant profile starting at random initial time. The first diagram provides the optimal feed extraction profiles with a similar meaning as in Figure 6. The last two diagrams plot the filling levels in the feed tank when applying these two extraction profiles to a set of 100 randomly generated inflow samples. Thick curves refer to the expected inflow profile. The second diagram (top right) indicates the corresponding probabilities of lower level violation as functions of time

\section{REFERENCES}

1. H. Arellano-Garcia, R. Henrion, P. Li, A. Möller, W. Römisch, M. Wendt, G. Wozny: A model for the online optimization of integrated distillation columns under stochastic constraints. DFG-Schwerpunktprogramm 'Echtzeit-Optimierung grosser Systeme', Preprint 98-32, 1998.

2. A. Barclay, P. E. Gill, J. B. Rosen: SQP Methods and their Application to Numerical Optimal Controll Report NA 97-3, Department of Mathematics, University of California, San Diego 1997.

3. E. Eich-Soellner, P. Lory, P. Burr, A. Kröner: Stationary and Dynamic Flowsheeting in the Chemical Engineering Industry. Surv. Math. Ind. 7 (1997), 1-28.

4. P. E. Gill, W. Murray, M. A. Saunders: SNOPT: An SQP Algorithm for Large-Scale Constrained Optimization. Report NA 97-2, Department of Mathematics, University of California, San Diego 1997.

5. P. E. Gill, W. Murray, M. A. Saunders: User's guide for SNOPT 5.3: A FORTRAN package for large-scale nonlinear programming. Report NA 97-2, Department of Mathematics, University of California, San Diego, 1997. 
6. J. Gmehling, U. Onken, W. Arlt: Vapour Liquid Equilibrium Data Collection, DECHEMA, Frankfurt, 1997.

7. E. Hairer, G. Wanner: Solving Ordinary Differential Equations II: Stiff and DifferentialAlgebraic Problems. Springer, Berlin, 1996.

8. R. Henrion, Structure and Stability of Probabilistic Storage Level Constraints. Preprint No. 23 (2000) of Stochastic Programming Eprint Series (SPEPS), submitted to: Proc. 4th GAMM/IFIP-Workshop on "STOCHASTIC OPTIMIZATION: Numerical Methods and Technical Applications", held at UniBw Munich, June 27-29, 2000.

9. R. Henrion, P. Li, A. Möller, M. C. Steinbach, M. Wendt, G. Wozny: Stochastic Optimization for Operating Chemnical Processes under Uncertainty. This volume.

10. R. Henrion, A. Möller: Optimization of a continuous distillation process under random inflow rate. Preprint No. 00-4 des DFG-Schwerpunktprogramms 'Echtzeit-Optimierung großer Systeme', Berlin, 2000, submitted to: Computers \& Mathematics with Applications.

11. H. Z. Kister: Distillation Design. McGraw-Hill, New York, 1992.

12. R. Lamour: A Well-Posed Shooting Method for Transferable DAE's. Numer. Math. 59 (1991), 815-829.

13. D. B. Leineweber, H. G. Bock and J. P. Schlöder: Fast Direct Methods for Real-Time Optimization of Chemical Processes. in: (A. Sydow ed.) Proceedings of the 15th IMACS World Congress on Scientific Computation, Modelling and Applied Mathematics 6, 1997, pp. 451-456.

14. R. C. Reid, J. M. Prausnitz, B. E. Poling: The Properties of Gases and Liquids. McGrawHill, New York, 1987.

15. A. Rix: Modellierung und Prozeßführung wärmeintegrierter Destillationskolonnen. $\mathrm{PhD}$ Thesis, TU Berlin, 1997.

16. V. Schulz: Reduced SQP Methods for Large-Scale Optimal Control Problems in DAE with Application to Path Planning Problems for Satellite Mounted Robots, Dissertation, Universität Heidelberg, 1995.

17. M. C. Steinbach: Fast Recursive SQP Methods for Large-Scale Optimal Control Problems, Dissertation, Universität Heidelberg, 1995.

18. O. von Stryk: Numerische Lösung optimaler Steuerungsprobleme: Diskretisierung, Parameteroptimierung und Berechnung der adjungierten Variablen, Dissertation, VDI Fortschritt-Berichte Reihe 8, Nr. 441, VDI-Verlag, Düsseldorf, 1995. 